\title{
Therapeutic potential of molecular hydrogen in ovarian cancer
}

\author{
Lei Shang $^{1,2 \#}$, Fei Xie ${ }^{1,2 \#}$, Jiala Li ${ }^{1,2}$, Yating Zhang ${ }^{1,2}$, Mengyu Liu ${ }^{1,2}$, Pengxiang Zhao ${ }^{1,2}$, Xuemei Ma ${ }^{1,2}$, Tyler W. \\ Lebaron $^{3}$
}

${ }^{1}$ College of Life Science and Bio-engineering, Beijing Molecular Hydrogen Research Center, Beijing University of Technology, Beijing 100124, China; ${ }^{2}$ Beijing Molecular Hydrogen Research Center, Beijing 100124, China; ${ }^{3}$ Molecular Hydrogen Institute, Kissimmee, FL, USA

Contributions: (I) Conception and design: X Ma, F Xie; (II) Administrative support: L Shang, J Li; (III) Provision of study materials or patients: M Liu; (IV) Collection and assembly of data: L Shang, J Li; (V) Data analysis and interpretation: L Shang, F Xie, P Zhao; (VI) Manuscript writing: All authors; (VII) Final approval of manuscript: All authors.

\#These authors contributed equally to this work.

Correspondence to: Xuemei Ma. College of Life Science and Bio-engineering, Beijing University of Technology, No.100, Pingleyuan, Chaoyang District, Beijing 100124, China. Email: xmma@bjut.edu.cn; Fei Xie. College of Life Science and Bio-engineering, Beijing University of Technology, No.100, Pingleyuan, Chaoyang District, Beijing 100124, China. Email: xiefei990815@bjut.edu.cn.

\begin{abstract}
Background: To investigate the potential effects of molecular hydrogen on ovarian cancer.
Methods: The in vivo study was performed using Hs38.T xenografted BALB/c nude mice. The mean tumor volume was monitored during 6 weeks of hydrogen inhalation. Immunohistochemistry (IHC) staining was performed to determine the Ki67 and CD34 expression. The in vitro effects of molecular hydrogen on proliferation of Hs38.T and PA-1 were determined by Cell Counting Kit (CCK)-8 assay. Matrigel invasion assay was performed to determine the cell invasion ability. Wounding assay was employed to examine the motile nature of ovarian cancer cells. Colony formation assay was performed to investigate the effect of molecular hydrogen in tumorigenicity. To further investigate the effects of molecular hydrogen on cancer stem cells (CSCs) properties, we performed sphere-formation assays.

Results: The in vivo study demonstrated that 6 weeks of hydrogen inhalation significantly inhibited tumor growth, as evidenced by decreased mean tumor volume (32.30\%) and Ki67 expression (30.00\%). Hydrogen treatment decreased the expression of CD34 (74.00\%) demonstrating its anti-angiogenesis effects. The in vitro study showed that hydrogen treatment significantly inhibits cancer cell proliferation, invasion, migration and colony formation both in Hs38.T and PA-1 cells. An important finding in this study was that molecular hydrogen could also markedly inhibit sphere-forming ability of both PA-1 and Hs38.T cells.

Conclusions: Molecular hydrogen may exert anti-tumor role in ovarian cancer through suppressing the proliferation of CSCs-like cells and angiogenesis.
\end{abstract}

Keywords: Ovarian cancer; molecular hydrogen; cancer stem cells-like cells (CSCs-like cells); angiogenesis

Submitted May 16, 2018. Accepted for publication Jul 11, 2018.

doi: $10.21037 /$ tcr.2018.07.09

View this article at: http://dx.doi.org/10.21037/tcr.2018.07.09

\section{Introduction}

Ovarian cancer is the fifth cause of cancer-related death in women and is characterized by an insidious onset and a lack of early specific symptoms (1). Ovarian cancer can be broadly classified into three major types on the basis of cellular origin, including epithelial cells (90\%), sexcord stromal cells (7\%) and germ cells (3-7\%) (2). The prevalent treatment of ovarian cancer consists of extensive surgery followed by chemotherapy. Although favorable results are achieved using Taxol and platinum-based chemotherapy, adverse side effects and acquired resistance are major impediments in ovarian cancer treatment. Despite continued research on treating ovarian cancer, its overall cure rate has remained at approximately $30 \%$ over the past two decades (3). Therefore, there is a great need to explore 
nontoxic, antitumor molecules for the treatment of ovarian cancer.

Molecular hydrogen has long been considered to be a physiologically inert gas. The biological relevance of molecular hydrogen has come a long way since Ohsawa et al. reported that hydrogen gas exerts a therapeutic antioxidant activity and suppresses brain damage caused by ischemia and reperfusion (4). Other scientists have also reported that molecular hydrogen has therapeutic effects in many diseases including ischemia-reperfusion (I/R) injuries, metabolic syndrome, neurodegeneration, mitochondrial diseases, inflammation, and cancer (5). Although the effects of molecular hydrogen are often ascribed to its antioxidant effect, the underlying molecular mechanisms remain elusive.

Oxidative stress has been associated with many diseases, including cancer. Although the potential effects of molecular hydrogen on cancer have been reported, these studies have been restricted to a limited number of cancer types, and/or only in vitro cell models. Dole et al. first reported the potential therapeutic role of molecular hydrogen on cutaneous squamous-cell carcinoma in 1975 (6). Molecular hydrogen has also been found to protect BALB/c mice from developing radiation-induced thymic lymphoma (7). Previous studies provide evidence that molecular hydrogen increases the survival rate of colon carcinoma-bearing mice, and inhibits lung cancer progression $(8,9)$. These findings suggest the potential therapeutic value of molecular hydrogen in the prevention and treatment of cancer.

The present study was performed to investigate the possible therapeutic effects of molecular hydrogen on ovarian cancer. Both in vivo and in vitro experimental models were used to evaluate the potential role of molecular hydrogen. We also investigated the mechanisms underlying the effects of molecular hydrogen on ovarian cancer.

\section{Methods}

\section{Animals and experimental design}

Female athymic nude BALB/c mice were purchased from Wei Tong Li Hua Company (Beijing, China). All of the animals were housed in pathogen-free conditions. All animal studies were conducted in accordance with the experimental protocols involving animal study approved by the Laboratory Animal Ethics Committee of Center of Functional Inspection of Health Food, College of Applied Science and Humanities, Beijing Union University. These mice were maintained under controlled conditions $\left(25^{\circ} \mathrm{C}\right.$,
$55 \%$ humidity and a $12 \mathrm{~h}$ day/night cycle) and fed standard laboratory food. For induction of subcutaneous ovarian tumor, $3 \times 10^{6} \mathrm{Hs} 38$. T cells in $0.1 \mathrm{~mL}$ of serum-free culture medium were injected subcutaneously into the left flank of mice. Two weeks later, the mice with ovarian tumors were randomly divided into two groups as follows: (I) mice in control group (control, $n=8$ ) were maintained under normal conditions; (II) mice in hydrogen treatment group ( $\mathrm{H}, \mathrm{n}=8)$ were treated with $\mathrm{H}_{2}$ inhalation $\left(33 \% \mathrm{H}_{2} / 66 \% \mathrm{O}_{2}\right)$ using a hydrogen-oxygen nebulizer machine (Shanghai Asclepius Meditec Corp., Shanghai, China) for $30 \mathrm{~min}$, three times per day. Tumor growth was monitored by measuring two bisecting diameters of each tumor with a caliper every 3 days during 6 weeks of hydrogen treatment. The tumor volume was calculated using the formula $\left(V=\mathrm{a} \times \mathrm{b}^{2} / 2\right)$, where a represents the largest diameter and $b$ the smallest diameter. At the end of the 6-week treatment mice were euthanized following their final $30 \mathrm{~min} \mathrm{H}_{2}$ treatment. Tumors of each group were completely removed, photographed, and fixed in 10\% formalin/PBS or stored in liquid nitrogen for histologic examination.

\section{Immunobistochemical examination of expression of Ki67 and $C D 34$}

The paraffin sections were deparaffinized in xylene and rehydrated with various concentrations of ethanol. To deactivate the endogenous oxidase, the sections were treated with $3 \% \mathrm{H}_{2} \mathrm{O}_{2}$ for $10 \mathrm{~min}$. The sections underwent antigen retrieval and blocking with goat serum. The specific primary antibody for Ki67 or CD34 (Abcam, Cambridge, MA, USA, $1: 300)$ was added to the sections and incubated overnight at $4{ }^{\circ} \mathrm{C}$ and then with horseradish peroxidase-conjugated secondary antibodies for $30 \mathrm{~min}$ at $37^{\circ} \mathrm{C}$. The sections were then developed with 3,3'-diaminobenzidine and counterstained with hematoxylin. Immunohistochemistry (IHC) staining intensity was measured by randomly selecting 3 high-power $(400 \times)$ fields per section using Image-Pro Plus software (Media Cybernetics, Rockville, MD, USA).

\section{Cell culture and bydrogen treatment}

Human ovarian cancer cell line Hs38.T and PA-1 were kindly provided by Beijing Cotimes Biotech Corp. and Cell Bank of Peking Union Medical College (PUMC, Beijing, China), cultured in Dulbecco's Modified Eagle's medium (DMEM) or Minimum Essential Medium with Earle's Balanced Salts (MEM/EBSS) containing 10\% fetal bovine 
serum (FBS) in a humidified atmosphere of $5 \% \mathrm{CO}_{2}$ at $37{ }^{\circ} \mathrm{C}$. Both Hs38.T and PA-1 cells were divided into two groups: (I) cells in control group (control) were cultured in normal complete medium; (II) cells in hydrogen treatment group (HM) were cultured in hydrogen-rich medium. The hydrogen-rich medium was prepared by placing a sterile plastic-shelled product consisting metallic magnesium (99.9\% pure) and natural stones in the polypropylene containers combined with ceramics (Doctor SUISOSUI ${ }^{\circledR}$; Friendear Inc., Tokyo, Japan) into DMEM medium containing 10\% FBS for more than $48 \mathrm{~h}$ before use at room temperature. The hydrogen concentration was monitored by using a needle-type Hydrogen Sensor (Unisense A/S, Aarhus, Denmark) every week. The stick was replaced every two weeks to ensure an $\mathrm{H}_{2}$ concentration of more than 600 $\mu \mathrm{M}$ was maintained.

\section{Cell viability analysis}

Cell viability was determined using a Cell Counting Kit (CCK)-8 assay (Dojindo Molecular Technologies Inc., Gaithersburg, MD, USA). Cells were plated in 96-well plates at a concentration of $5.0 \times 10^{3} /$ well and cultured for $24 \mathrm{~h}$. To develop growth curves, cells were further cultured for $0,24,48,72,96$, and $120 \mathrm{~h}$ in normal complete medium or hydrogen-rich medium. After incubation, $10 \mu \mathrm{L}$ of CCK-8 solution was added to each well and incubated for $3 \mathrm{~h}$ at $37^{\circ} \mathrm{C}$. The absorbance value of each well was read immediately at $450 \mathrm{~nm}$ using a microplate reader (Bio-Rad, Richmond, CA, USA).

\section{Cell invasion assay}

The cell invasion ability was determined using BD Matrigel invasion chambers according to the manufacturer's protocol. Briefly, the top chambers with polycarbonate filters ( $8 \mu \mathrm{m}$ pore size; Costar, Acton, MA, USA) were coated with $50 \mu \mathrm{L}$ of Matrigel $\left(0.8 \mu \mathrm{g} / \mu \mathrm{L}, 37^{\circ} \mathrm{C}, 2 \mathrm{~h}\right.$; BD Biosciences, San Diego, CA). $1 \times 10^{5}$ cells in $100 \mu \mathrm{L}$ serum-free medium were seeded to the top chamber and $650 \mu \mathrm{L}$ normal medium or hydrogen-rich medium was added to the bottom. The cells were allowed to migrate through the porous membrane at $37^{\circ} \mathrm{C}$ for $48 \mathrm{~h}$. Cells in the upper surface of the chamber were completely removed by cotton swabs. The cells on the lower surface were stained with $0.1 \%(\mathrm{w} / \mathrm{v})$ crystal violet after fixation, and five random fields from each insert were counted at $100 \times$ magnifications. The invasion assay was conducted in triplicate independent experiments.

\section{Cell migration assay}

Cell migration assay was carried out using a woundhealing assay. Briefly, cells were seeded in 6-well plates at a concentration of $1.0 \times 10^{6} \%$ well and cultured for $24 \mathrm{~h}$. A plastic pipette tip was used to scratch a line across the cell surface in each plate. The remaining cells were washed three times with PBS to remove the floating cells and debris, followed by incubation for $48 \mathrm{~h}$ in normal complete medium or hydrogen-rich medium. The images of the healing process were photographed digitally at the time point of $0,24 \mathrm{~h}$ after wounding. The wound-healing assay was performed in three independent experiments.

\section{Colony formation assay}

One thousand cells per well were counted and seeded in 6 -well plates. The plates were incubated for 14 days in normal or hydrogen-rich medium. The cells were then fixed by $4 \%$ paraformaldehyde and stained using $0.1 \%$ crystal violet. Colonies were counted only if they included at least 15 cells. Triplicate independent experiments were performed and all the visible colonies were calculated manually.

\section{Sphere formation assay}

Cells were collected and washed to remove serum, then suspended in serum-free DMEM or MEM/EBSS medium at a density of $2.0 \times 10^{3} / \mathrm{mL}$. Five hundred cells per well were counted and seeded onto ultra-low attachment 6-well plates (Corning, Corning, NY, USA) for the sphere-formation assay. Cells were cultured in normal serum-free medium or hydrogen-rich serum-free medium with B27 supplement (GIBCO, Grand Island, NY, USA), $20 \mathrm{ng} / \mathrm{mL}$ of epidermal growth factor (EGF) (Pepro Tech Inc., Rocky Hill, NJ, USA), and $20 \mathrm{ng} / \mathrm{mL}$ of basic fibroblast growth factor (bFGF) (Pepro Tech). Medium was changed every 3 days. Cells were incubated for 10 days, and spheres with diameter $>50 \mu \mathrm{m}$ were counted.

\section{Statistical analysis}

Groups from cell culture and in vivo experiments were compared using two-tailed Student's $t$-tests and results were presented as means $\pm \mathrm{SD}$. All statistical analyses were performed using GraphPad Prism 6.01. A value of $\mathrm{P}<0.05$ was considered significant. 
A

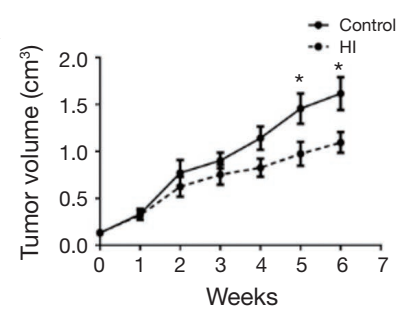

C

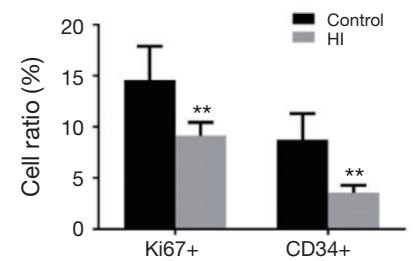

B

Ki67

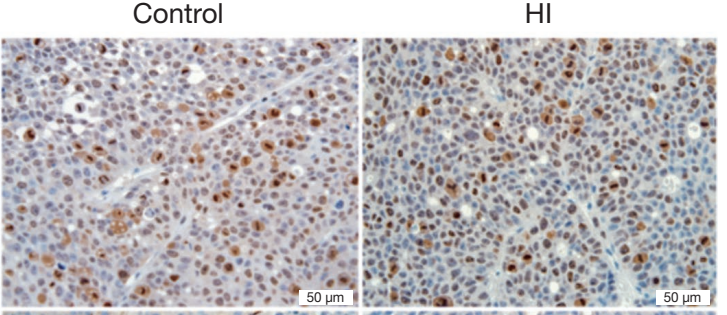

$\mathrm{CD} 34$

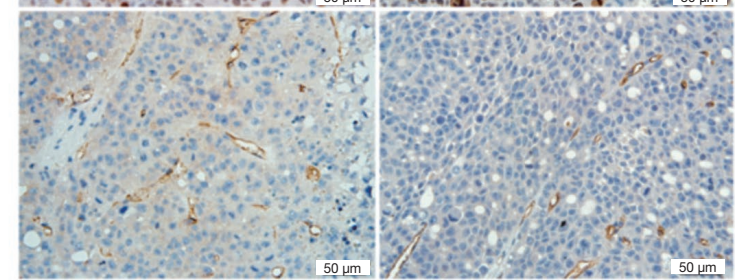

Figure 1 Hydrogen inhalation inhibited the tumor growth and angiogenesis in Hs38.T xenografted BALB/c nude mice. (A) Six weeks of hydrogen inhalation induced a significant decrease in mean tumor volume; (B) immunohistochemical examination showed that the expression of Ki67 and CD34 were down-regulated by molecular hydrogen; (C) quantification of immunohistochemical staining. *, $\mathrm{P}<0.05$; **, $\mathrm{P}<0.01$. HI, hydrogen inhalation.

\section{Results}

\section{Molecular hydrogen suppresses tumor growth in vivo}

To address the impact of molecular hydrogen on ovarian cancer biology in vivo, Hs38.T cells were xenografted onto nude mice and treated with hydrogen for 6 weeks. As shown in Figure $1 A$, the difference in mean tumor volume reached statistical significance at week 5 , a marked reduction after hydrogen treatment was observed at week $5(33.06 \%, \mathrm{P}=0.0475)$ and week $6(32.30 \%, \mathrm{P}=0.0347)$ compared with the controls.

\section{Effects of molecular hydrogen on Ki67 and CD34 expression}

IHC analysis with Ki67 staining demonstrated that the percentage of Ki67-positive proliferating cells was 30.00\% lower in hydrogen group than that in controls (Figure 1B,C). Angiogenesis was evaluated by CD34 immunohistochemistry and the results showed that the ratio of CD34-positive cell in hydrogen group was significantly decreased by $74.00 \%$ compared to the controls (Figure 1B,C).

\section{Effects of molecular hydrogen on cell growth, invasion and migration}

In vitro effects of molecular hydrogen on proliferation of human ovarian cancer cells were determined by CCK- 8 assay. The results show that hydrogen treatment induced a slightly slower growth rate in PA-1 cells and the maximal effect on proliferation inhibition was observed (12.97\%) at the 24 h time point (Figure $2 A$ ). Similarly, there was a decreased growth rate in Hs38.T cells after hydrogen treatment. Molecular hydrogen significantly inhibited cell proliferation at the 48 and $96 \mathrm{~h}$ time point by $22.79 \%$ and $17.26 \%$, respectively (Figure $2 B$ ).

In parallel, a Matrigel invasion assay was also performed to determine the effect of hydrogen treatment on the invasion of human ovarian cancer cells. As shown in Figure 2C,D, hydrogen treatment induced a significant decrease in cell invasiveness in both Hs38.T and PA-1 cells (39.28\%, $\mathrm{P}=0.0284$; and $72.36 \%, \mathrm{P}=0.0005$ ).

In vitro wounding assay was employed to examine the influence of hydrogen treatment on the migration of human ovarian cancer cells. As shown in Figure 2E,F, the presence of hydrogen significantly inhibited the motile nature in both PA- 1 and Hs38.T cells by $20.32 \%(P=0.0136)$ and $38.96 \%$ ( $\mathrm{P}=0.0307)$, respectively.

\section{Effects of molecular hydrogen on clonogenicity in vitro}

To investigate the potential role of molecular hydrogen in tumorigenicity, in vitro colony formation assay was performed. The results showed that hydrogen treatment induced markedly fewer and smaller colonies in both Hs38.T and PA-1 cells. As shown in Figure 3, hydrogen treatment significantly decreased colony numbers of PA-1 (45.11\%, $\mathrm{P}=0.0015)$ and $\mathrm{Hs} 38$. T cells $(19.70 \%, \mathrm{P}=0.0021)$. There was also a marked reduction in diameter of colonies after hydrogen treatment in both PA-1 $(31.08 \%, \mathrm{P}=0.0002)$ and 

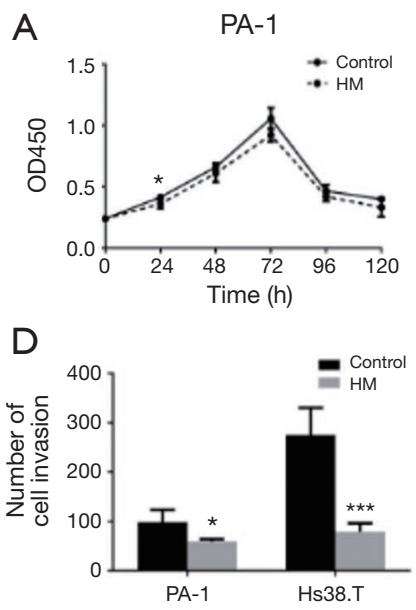

$\mathrm{F}$

Control

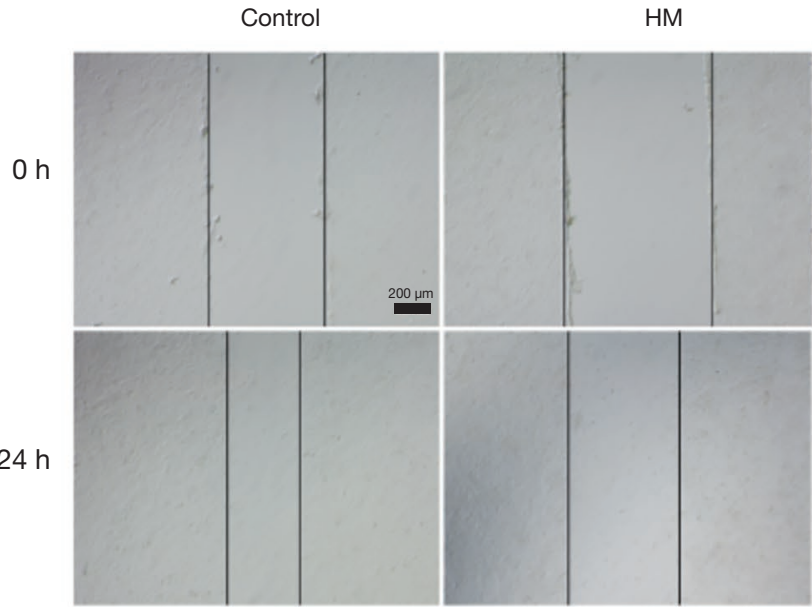

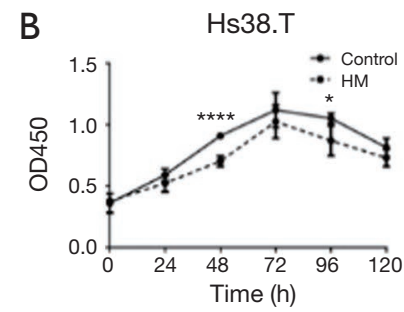

$E$

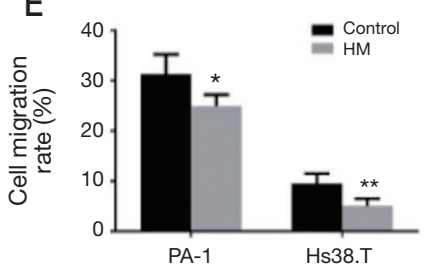

PA-1

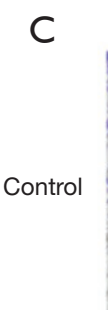

HM

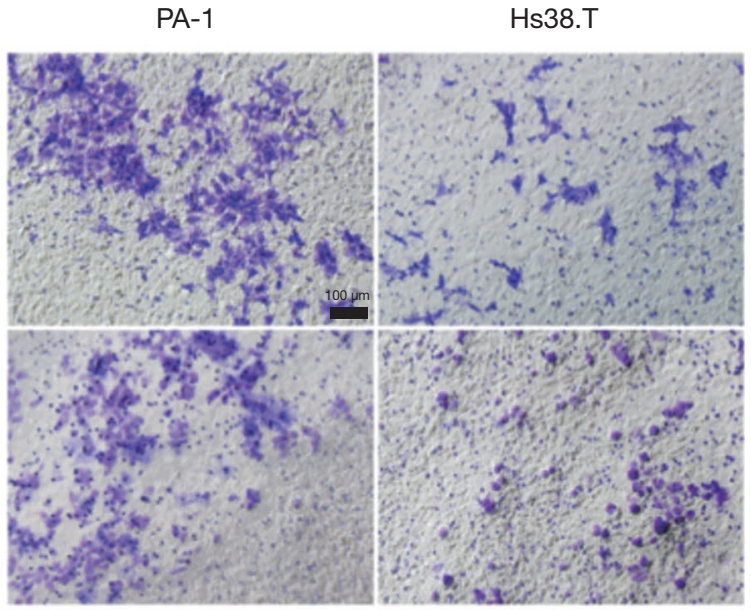

Hs38.T

Control $\quad$ HM

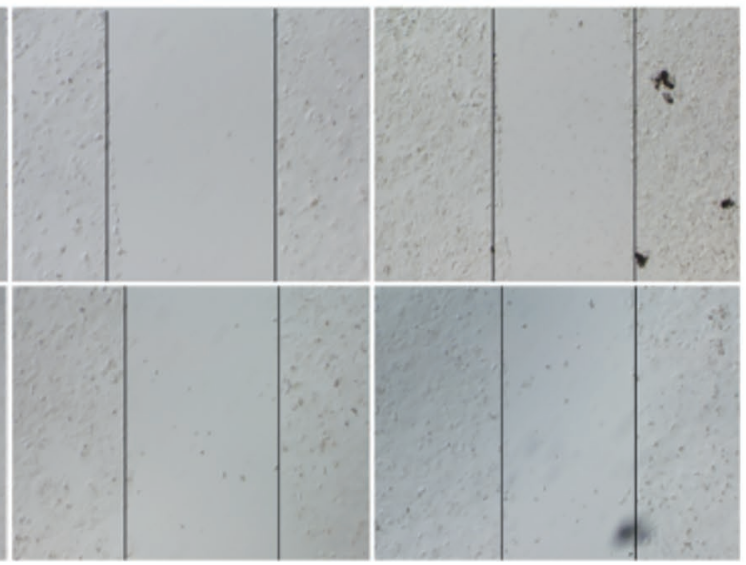

Figure 2 The effects of molecular hydrogen on the cell function of human ovarian cancer cells. (A,B) CCK-8 assay demonstrated that the cell proliferation was inhibited by hydrogen treatment in PA-1 and Hs38.T cells; (C,D) Matrigel invasion assay showed that hydrogen treatment significantly inhibits the cell invasion in both PA-1 and Hs38.T cells; (E,F) wound-healing assay indicated that the cell migration of PA-1 and Hs38.T cells were inhibited by hydrogen. * $\mathrm{P}<0.05$; **, $\mathrm{P}<0.01$; ${ }^{* * *}, \mathrm{P}<0.001$; ${ }^{* * *}, \mathrm{P}<0.0001$. CCK-8, Cell Counting Kit-8, HM, hydrogen-rich medium.

Hs38.T cells $(38.33 \%, \mathrm{P}<0.0001)$.

\section{Effects of molecular hydrogen on sphere formation}

To further investigate the effects of molecular hydrogen on cancer stem cells (CSC) properties, we performed sphere formation assays. As shown in Figure 4, although the slight reduction of the floating sphere number of Hs38.T cells by molecular hydrogen was not statistically significant, hydrogen did significantly decrease the average diameter of floating sphere by $41.48 \%(\mathrm{P}<0.0001)$. Additionally, in the PA-1 cells, hydrogen treatment led to a marked reduction of sphere number $(82.47 \%$, $\mathrm{P}=0.0006)$ and average diameter $(55.25 \%, \mathrm{P}<0.0001)$.

\section{Discussion}

Although the therapeutic effects of molecular hydrogen have been investigated in a wide range of disease models and human diseases, there is only a paucity of reports on the anti-tumor effect of molecular hydrogen. To the best of our knowledge, this is the first report providing in vivo and in vitro evidence demonstrating the potential therapeutic 

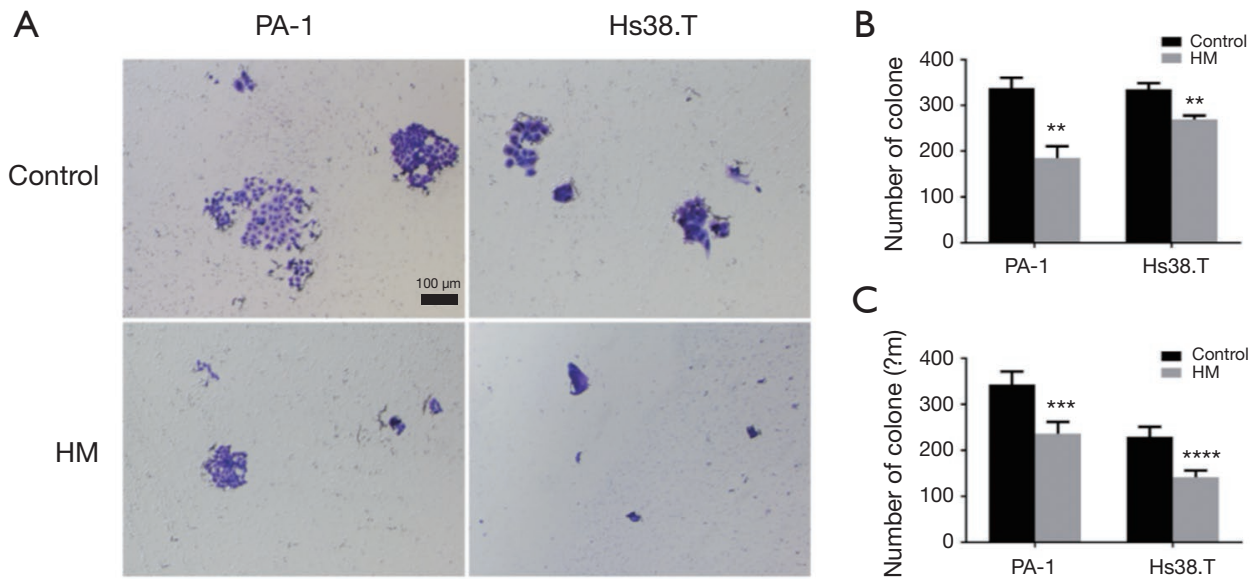

Figure 3 The inhibitory effect of molecular hydrogen on clonogenicity in human ovarian cancer cells. (A) Representative images showed the inhibitory effect of molecular hydrogen on colony formation; (B) the number of colonies was significantly decreased by hydrogen treatment in both PA-1 and Hs38.T cells; (C) the average diameter of colonies in both PA-1 and Hs38.T cells was markedly reduced in hydrogen group than that of controls. ${ }^{* *}, \mathrm{P}<0.01 ;{ }^{* * *}, \mathrm{P}<0.001 ;{ }^{* * *}, \mathrm{P}<0.0001$. HM, hydrogen-rich medium.

A

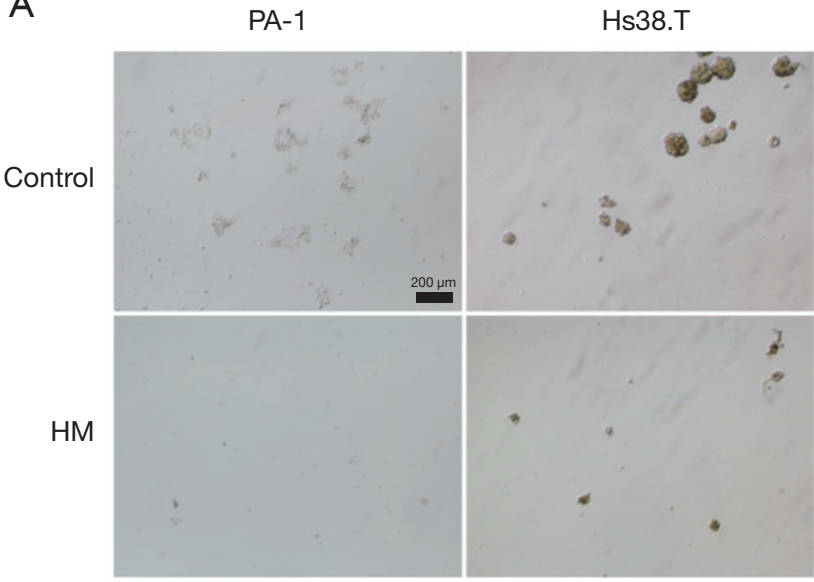

B

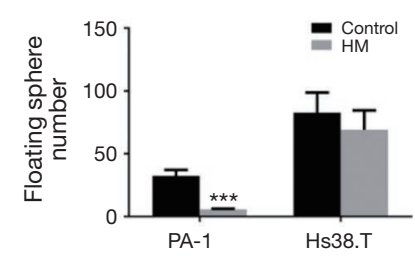

C

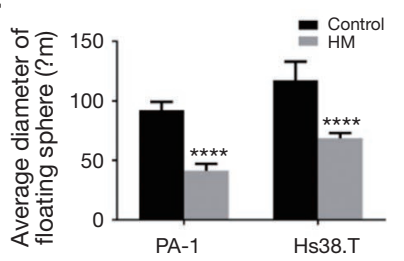

Figure 4 The inhibitory effect of molecular hydrogen on sphere formation in human ovarian cancer cells. (A) Representative images showed the inhibitory effect of molecular hydrogen on sphere formation; (B) the floating sphere number of PA-1 cells was significantly decreased by hydrogen treatment, while no marked change was observed in Hs38.T cells; (C) the average diameter of floating sphere in both Hs38.T and PA-1 cells was markedly reduced in hydrogen group than that of controls. ${ }^{* * *}, \mathrm{P}<0.001 ;{ }^{* * *}, \mathrm{P}<0.0001$. HM, hydrogen-rich medium.

role of molecular hydrogen on ovarian cancer. Our in vivo study demonstrated that hydrogen inhalation for 6 weeks significantly suppressed tumor growth in a mouse model of ovarian cancer. This anti-tumor effect of molecular hydrogen was also confirmed at the cellular level. The in vitro study showed that hydrogen treatment significantly inhibited cell proliferation, invasion, migration, colony formation, and sphere formation in both Hs38.T and PA-1 cells. These findings suggest the clinical potential of molecular hydrogen for preventive and therapeutic anti-tumor applications.

Since 2007, when Ohsawa et al. reported that inhalation of $2 \%$ hydrogen gas can protect the brain against I/R injury and stroke (4), most research on the biological effects of molecular hydrogen have focused on the inhalation of low concentration of hydrogen gas (1-4\%) or normobaric hydrogen. Hyperbaric hydrogen or high concentration of hydrogen may be effective for some diseases, but only a limited number of studies have been published. Dole et al. 
first reported an anti-cancer effect of hydrogen in Science in 1975 (6). They found that inhalation of $2.5 \%$ oxygen and $97.5 \%$ hydrogen under 8 -atmospheric pressure exhibits prominent suppression on squamous cell carcinoma. A recent study showed that inhalation of $60 \%$ hydrogen gas for 4 weeks in a xenografted model in nude mice could inhibit the carcinogenesis in lung cancer. This in vitro study indicated that a higher concentration of hydrogen may have a better anti-tumor effect (9). Our study showed that inhalation of $66.7 \%$ hydrogen gas could exert an anti-tumor role in ovarian cancer. These results indicate that high concentration of hydrogen may be more effective on the treatment of cancer, although the dose-effect relationship of hydrogen needs to be further investigated.

There are three methods most often used to administer hydrogen, including inhalation of hydrogen gas, drinking hydrogen-rich water, and injection of hydrogen-rich saline. Inhalation of hydrogen gas increases the likelihood that $\mathrm{H}_{2}$ molecules arrived at the actual cancerous tissues at high concentrations, which may not be the case with drinking or injection. The in vivo study inhaled hydrogen for $30 \mathrm{~min}$, which may be enough to start to reach an equilibrium. At $66.7 \% \mathrm{H}_{2}$, the hydrogen concentration would theoretically be at around $520 \mu \mathrm{M}$, which is comparable to the in vitro study of $600 \mu \mathrm{M} \mathrm{H}_{2}$. In addition, mice inhaled a higher oxygen concentration (33.3\%); consequently, the presence of increased oxygen may also have an inhibitory effect on cancer growth (10).

In the current study, 6 weeks of hydrogen inhalation significantly inhibited ovarian cancer growth. This was further confirmed by IHC examination, which showed that molecular hydrogen down-regulated the expression of the proliferation marker Ki67. The in vitro study also confirmed the inhibitory effect of molecular hydrogen on ovarian cancer, as evidenced by the reduced cell proliferation in both Hs38.T and PA-1 cells. Previous studies have also provided evidence for the inhibitory effect of molecular hydrogen on cancer. Saitoh et al. reported that molecular hydrogen caused growth inhibition of human tongue carcinoma cells HSC-4 and human fibrosarcoma cells HT-1080 but did not compromise growth of normal human tongue epithelial-like cells DOK (11). In another report, Kawai et al. established a streptozotocininduced nonalcoholic steatohepatitis (NASH)-related hepatocarcinogenic mouse model and found that drinking hydrogen-rich water for 8 weeks could improve NASH-related hepatocarcinogenesis, as evidenced by the reduction of both tumor number and tumor size; however, this study did not provide evidence for the inhibitory effect on tumor growth (12).

Recent studies suggest that malignant tumors can be seen as abnormal organs containing a small subset of CSCs with the capacity to self-renew, differentiate, and generate a new tumor (13). These CSCs are responsible for sustaining tumor growth, metastasis and drug resistance (14). CSCs behave as stem cells in that they can self-renew and differentiate into heterogeneous tumor cells. PA- 1 cell line is an excellent model for CSCs because it abundantly expresses the putative CSCs marker CD133 $(15,16)$. In addition to using biomarkers to distinguish CSCs, analysis of capacity for sphere formation is also a reliable method for detecting and characterizing CSCs populations (16). An important finding in this study was that molecular hydrogen could markedly inhibit the sphere-forming ability of both PA-1 and Hs38.T cells. These data suggest that the CSCs-like cells in ovarian cancer may be more susceptible to hydrogen treatment, and the decreases in CSCs-like cells may result in the decreased cell proliferation in total ovarian cancer cells.

Angiogenesis is a pathophysiological process involving the growth of new blood vessels from pre-existing capillaries, excessive and abnormal vasculature, which contributes to the progression of solid tumors (17). An important finding in this study was that molecular hydrogen could suppress angiogenesis in ovarian cancer, as evidenced by decreased expression of CD34 in hydrogen-treated mice. This anti-angiogenesis effect was also observed by a previous report. Ye et al. demonstrated that molecular hydrogen can suppress angiogenesis induced by A549 cells through down-regulating both $\mathrm{H}_{2} \mathrm{O}_{2}$ release and VEGF expression (18). However, some of the results in the study by Ye et al. may have also been attributed to PtNPs from electrolysis. These findings suggest that molecular hydrogen may exert its anti-tumor role by the inhibition of angiogenesis; however, the underlying molecular mechanisms require further elucidation.

We also examined the effect of molecular hydrogen on the migration and invasion of ovarian cancer cells. The results show that molecular hydrogen could suppress cell migration in both PA-1 and Hs38.T cells. The repressive effect of molecular hydrogen on cell invasion of HT-1080 cells was reported in previous study (11), and this effect was observed in both Hs38. $\mathrm{T}$ and PA- 1 cells. Taken together, these data suggest that molecular hydrogen may exert its anti-tumor effect in ovarian cancer through the regulation of cell invasion and migration.

In conclusion, the present study demonstrates that inhalation of $66.7 \%$ molecular hydrogen has an inhibitory effect on ovarian cancer growth. Moreover, molecular hydrogen also suppresses the proliferation of CSCs-like cells and angiogenesis, which may contribute to its anti- 
tumor effect. Therefore, our results suggest that molecular hydrogen potentially be used for treating ovarian cancer.

\section{Acknowledgments}

Funding: This study was supported by National Natural Science Foundation of China (grant number: 31500828).

\section{Footnote}

Conflicts of Interest: All authors have completed the ICMJE uniform disclosure form (available at http://dx.doi. org/10.21037/tcr.2018.07.09). The authors have no conflicts of interest to declare.

Ethical Statement: The authors are accountable for all aspects of the work in ensuring that questions related to the accuracy or integrity of any part of the work are appropriately investigated and resolved. Use of animals was approved by the institutional ethical committee following the National Research Council Guide the Care and Use of Laboratory Animals. The ethical approval number is 2016 S012.

Open Access Statement: This is an Open Access article distributed in accordance with the Creative Commons Attribution-NonCommercial-NoDerivs 4.0 International License (CC BY-NC-ND 4.0), which permits the noncommercial replication and distribution of the article with the strict proviso that no changes or edits are made and the original work is properly cited (including links to both the formal publication through the relevant DOI and the license). See: https://creativecommons.org/licenses/by-nc-nd/4.0/.

\section{References}

1. Karnezis AN, Cho KR, Gilks CB, et al. The disparate origins of ovarian cancers: pathogenesis and prevention strategies. Nat Rev Cancer 2017;17:65-74.

2. Shanmugasundaram G, Sundaramoorthy E, Sudalaiandi S, et al. Double Pathology: Malignant Epithelial Ovarian Tumor and Germ Cell Tumor (Choriocarcinoma), a Rare Coexistence. World J Oncol 2015;6:421-5.

3. Bast RC Jr, Hennessy B, Mills GB. The biology of ovarian cancer: new opportunities for translation. Nat Rev Cancer 2009;9:415-28.

4. Ohsawa I, Ishikawa M, Takahashi K, et al. Hydrogen acts as a therapeutic antioxidant by selectively reducing cytotoxic oxygen radicals. Nat Med 2007;13:688-94.
5. Ohta S. Molecular hydrogen as a preventive and therapeutic medical gas: initiation, development and potential of hydrogen medicine. Pharmacol Ther 2014;144:1-11.

6. Dole M, Wilson FR, Fife WP. Hyperbaric hydrogen therapy: a possible treatment for cancer. Science 1975;190:152-4.

7. Zhao L, Zhou C, Zhang J, et al. Hydrogen protects mice from radiation induced thymic lymphoma in BALB/c mice. Int J Biol Sci 2011;7:297-300.

8. Runtuwene J, Amitani H, Amitani M, et al. Hydrogenwater enhances 5 -fluorouracil-induced inhibition of colon cancer. Peer J 2015;3:e859.

9. Wang $\mathrm{D}$, Wang $\mathrm{L}$, Zhang $\mathrm{Y}$, et al. Hydrogen gas inhibits lung cancer progression through targeting SMC3. Biomed Pharmacother 2018;104:788-97.

10. Moen I, Stuhr LE. Hyperbaric oxygen therapy and cancer-a review. Target Oncol 2012;7:233-42.

11. Saitoh Y, Okayasu H, Xiao L, et al. Neutral pH hydrogenenriched electrolyzed water achieves tumor-preferential clonal growth inhibition over normal cells and tumor invasion inhibition concurrently with intracellular oxidant repression. Oncol Res 2008;17:247-55.

12. Kawai D, Takaki A, Nakatsuka A, et al. Hydrogen-rich water prevents progression of nonalcoholic steatohepatitis and accompanying hepatocarcinogenesis in mice. Hepatology 2012;56:912-21.

13. Visvader JE, Lindeman GJ. Cancer stem cells: current status and evolving complexities. Cell stem cell 2012;10:717-28.

14. Zhou BB, Zhang H, Damelin M, et al. Tumour-initiating cells: challenges and opportunities for anticancer drug discovery. Nat Rev Drug Discov 2009;8:806-23.

15. Skubitz AP, Taras EP, Boylan KL, et al. Targeting CD133 in an in vivo ovarian cancer model reduces ovarian cancer progression. Gynecol Oncol 2013;130:579-87.

16. Chung WM, Chang WC, Chen L, et al. MicroRNA-21 promotes the ovarian teratocarcinoma PA1 cell line by sustaining cancer stem/progenitor populations in vitro. Stem Cell Res Ther 2013;4:88.

17. Folkman J. Tumor angiogenesis: therapeutic implications. N Engl J Med 1971;285:1182-6.

18. Ye J, Li Y, Hamasaki T, et al. Inhibitory effect of electrolyzed reduced water on tumor angiogenesis. Biol Pharm Bull 2008;31:19-26.

Cite this article as: Shang L, Xie F, Li J, Zhang Y, Liu M, Zhao P, Ma X, Lebaron TW. Therapeutic potential of molecular hydrogen in ovarian cancer. Transl Cancer Res 2018;7(4):988-995. doi: 10.21037/tcr.2018.07.09 